\title{
EULER CHARACTERISTIC OF COHERENT SHEAVES ON SIMPLICIAL TORICS VIA THE STANLEY-REISNER RING
}

\author{
HAL SCHENCK
}

\begin{abstract}
We combine work of Cox on the total coordinate ring of a toric variety and results of Eisenbud-Mustaţă-Stillman and Mustață on cohomology of toric and monomial ideals to obtain a formula for computing $\chi\left(\mathcal{O}_{X}(D)\right)$ for a Weil divisor $D$ on a complete simplicial toric variety $X_{\Sigma}$. The main point is to use Alexander duality to pass from the toric irrelevant ideal, which appears in the computation of $\chi\left(\mathcal{O}_{X}(D)\right)$, to the Stanley-Reisner ideal of $\Sigma$, which is used in defining the Chow ring of $X_{\Sigma}$.
\end{abstract}

\section{INTRODUCTION}

For a divisor $D$ on a smooth complete variety $X$, the Hirzebruch-Riemann-Roch theorem describes the Euler characteristic of $\mathcal{O}_{X}(D)$ in terms of intersection theory:

$$
\chi\left(\mathcal{O}_{X}(D)\right)=\int \operatorname{ch}(D) \cdot \operatorname{Td}(X) .
$$

The divisor $D$ corresponds to a class $[D]$ in the Chow ring of $X$, and $\operatorname{ch}(D)$ consists of the first $n=\operatorname{dim}(X)$ terms of the formal Taylor expansion of $e^{D}$. The Todd class of $D$ is defined similarly, but using the Taylor expansion for $\frac{D}{1-e^{-D}}$. To define the Todd class of $X$, filter the tangent bundle $\mathcal{T}_{X}$ by line bundles $\mathcal{O}\left(D_{i}\right)$. Then one shows that $T d(X)=\prod_{i=1}^{n} T d\left(D_{i}\right)$ is independent of the filtration.

Let $\Sigma \subseteq \mathbb{R}^{n}$ be a complete simplicial rational polyhedral fan with $d=|\Sigma(1)|$ rays, $X_{\Sigma}$ the associated toric variety, and $D \in \mathrm{Cl}\left(X_{\Sigma}\right)$ a Weil divisor on $X_{\Sigma}$. We combine Alexander duality and the Cox ring with results of Mustaţă [16] on monomial ideals to obtain a formula for the Euler characteristic of the associated rank one reflexive sheaf $\mathcal{O}_{X_{\Sigma}}(D)$. Put $Z=\{0,1\}^{d}$ and $\mathbf{1}=\{1\}^{d}$. Then for $l \gg 0$,

$$
\chi\left(\mathcal{O}_{X}(D)\right)=\sum_{\mathbf{m} \in Z \backslash \mathbf{0}}(-1)^{|\mathbf{m}|-d+n} \operatorname{dim}_{\mathbb{K}}\left(S / I_{\Sigma}\right)_{\mathbf{1}-\mathbf{m}} \cdot \operatorname{dim}_{\mathbb{K}} S_{l \cdot \phi(\mathbf{m})+D} .
$$

Here $I_{\Sigma}$ denotes the Stanley-Reisner ideal, and $\mathbb{Z}^{d} \stackrel{\phi}{\rightarrow} \mathrm{Cl}\left(X_{\Sigma}\right)$ is the standard surjection of $\mathbb{Z}^{d}$ onto the class group. The Cox ring $S$ is a polynomial ring, graded by $\mathrm{Cl}\left(X_{\Sigma}\right)$; on $S / I_{\Sigma}$ we use the $\mathbb{Z}^{d}$ grading. We recall the definitions of these objects in $\S 2$. Any coherent sheaf on a nondegenerate toric variety corresponds to a finitely generated $\mathrm{Cl}\left(X_{\Sigma}\right)$-graded $S$-module (see 3 for the simplicial case, and 17] for the general case), so such a sheaf has a resolution by rank one reflexive sheaves, and Equation 1 yields a formula for $\chi(\mathcal{F})$ for any coherent sheaf $\mathcal{F}$. Bounds on $l$ are determined by Eisenbud-Mustaţă-Stillman in [], and are discussed in $\S 2$.

2000 Mathematics Subject Classification. 14M25, 14C40.

Key words and phrases. Toric variety, Cox ring, Chow ring, cohomology.

Schenck supported by NSF 07-07667, NSA 904-03-1-0006. 
Connections to physics and some history. The methods which are used to prove Equation 10 have applications to computations arising in mathematical physics: in a recent preprint [1, Blumenhagen, Jurke, Rahn and Roschy conjectured an algorithm for computing the cohomology of line bundles on a toric variety. Their motivation was to compute massless modes in Type IIB/F and heterotic compactifications, on a complete intersection in a toric variety. A strong form of the algorithm is established by Maclagan and Smith in Corollary 3.4 of [14]; later proofs appear in Jow [9] and Rahn-Roschy [20]. In all these papers Alexander duality and results of [6] play a key role, as they do in the proof of Equation 11. The original motivation for this work was to find a toric proof for the Hirzebruch-Riemann-Roch theorem.

The first toric interpretation of Hirzebruch-Riemann-Roch is due to Khovanskii [11. In [12, [13, Pukhlikov-Khovanskii study additive measures on virtual polyhedra, and obtain a Riemann-Roch formula for integrating sums of quasipolynomials on virtual polytopes. Pommersheim [18] and Pommersheim and Thomas [19] obtain results on Todd classes of simplicial torics, and in [2], Brion-Vergne prove an equivariant Riemann-Roch for simplicial torics.

The results of Eisenbud-Mustaţă-Stillman in 6 show that in the toric setting, $\chi\left(\mathcal{O}_{X}(D)\right)$ may be calculated via certain Ext modules over the Cox ring of $X$. On the other hand, evaluating the expression $\int \operatorname{ch}(D) \cdot T d(X)$ involves a computation in the Chow ring of $X$, and the Cox and Chow rings of a simplicial toric variety are connected by Alexander duality.

The paper is structured as follows: in $\S 2$ we recall the results of [6] and the computation of cohomology via the Cox ring. In $\S 3$ we introduce the Chow ring, recall that the Stanley-Reisner ideal of $\Sigma$ is the Alexander dual of the toric irrelevant ideal of $\Sigma$, and use results of Mustaţă and Stanley to connect the parts. Equation 1 is proved in $\S 4$, and illustrated on the Hirzebruch surface $\mathcal{H}_{2}$.

Toric facts. Let $N \simeq \mathbb{Z}^{n}$ be a lattice, with dual lattice $M$, and let $\Sigma \subseteq N \otimes_{\mathbb{Z}} \mathbb{R} \simeq$ $\mathbb{R}^{n}$ be a complete simplicial rational polyhedral fan (henceforth, simply fan), with $\Sigma(i)$ denoting the set of $i$-dimensional faces of $\Sigma$, and let $X_{\Sigma}$ be the associated toric variety. A Weil divisor on $X_{\Sigma}$ is of the form

$$
D=\sum_{\rho \in \Sigma(1)} a_{\rho} D_{\rho}, \text { with } a_{\rho} \in \mathbb{Z} .
$$

Let $d=|\Sigma(1)|$. The class group of $X_{\Sigma}$ has a presentation

$$
0 \longrightarrow M \stackrel{\psi}{\longrightarrow} \mathbb{Z}^{d} \stackrel{\phi}{\longrightarrow} \mathrm{Cl}\left(X_{\Sigma}\right) \longrightarrow 0,
$$

where $\psi$ is defined by

$$
\chi^{m} \mapsto \sum_{\rho \in \Sigma(1)}\left\langle m, v_{\rho}\right\rangle D_{\rho}, \text { where } v_{\rho} \text { is a minimal lattice generator for } \rho .
$$

In 3], Cox introduced the total coordinate ring (henceforth called the Cox ring) of $X_{\Sigma}$. This is a polynomial ring, graded by the class group $\mathrm{Cl}\left(X_{\Sigma}\right)$.

\section{Definition 1.1.}

$$
S=\mathbb{K}\left[x_{\rho} \mid \rho \in \Sigma(1)\right]=\bigoplus_{\alpha \in \operatorname{Cl}\left(X_{\Sigma}\right)} S_{\alpha}
$$

The utility of this grading is that for $\alpha \simeq D \in \mathrm{Cl}\left(X_{\Sigma}\right), H^{0}\left(\mathcal{O}_{X}(D)\right) \simeq S_{\alpha}$. For more background on toric varieties, see [4, [5], or [7]. 


\section{Cohomology And the Cox Ring}

The Cox ring has a distinguished ideal, the toric irrelevant ideal

$$
B(\Sigma)=\left\langle x^{\hat{\sigma}} \mid \sigma \in \Sigma\right\rangle \text {, where } x^{\hat{\sigma}}=\prod_{\rho \notin \sigma(1)} x_{\rho} .
$$

Note that $B(\Sigma)$ is generated by monomials corresponding to the complements of the maximal faces of $\Sigma$. For an ideal $I=\left\langle f_{1}, \ldots, f_{m}\right\rangle$ let

$$
I^{[l]}=\left\langle f_{1}^{l}, \ldots, f_{m}^{l}\right\rangle \text {. }
$$

In 6], Eisenbud-Mustaţă-Stillman show that for $D \in \mathrm{Cl}\left(X_{\Sigma}\right), i \geq 1$ and $l \gg 0$,

$$
H^{i}\left(\mathcal{O}_{X}(D)\right) \simeq \operatorname{Ext}_{S}^{i+1}\left(S / B(\Sigma)^{[l]}, S(D)\right)_{0},
$$

They also obtain a bound for $l$. Fix a basis for $M$, and let $A$ be a $d \times n$ matrix with a row for each ray $u_{\rho} \in \Sigma(1)$, written with respect to the fixed basis. Define

$$
\begin{aligned}
a & =\max (\mid \text { entries of } A \mid) \\
b & =\max (\mid(n-1) \times(n-1) \text { minors of } A \mid) \\
c & =\min (\mid \text { nonzero } n \times n \text { minors of } A \mid) .
\end{aligned}
$$

Corollary 3.3 of [6] shows that if $D=\sum_{\rho} a_{\rho} D_{\rho}$, then Equation 2 holds for

$$
l \geq n^{2} \max _{\rho \in \Sigma(1)}\left(\left|a_{\rho}\right|\right) a b / c
$$

For brevity, we use lower case to denote $\operatorname{dim}_{\mathbb{K}}$ of an object, e.g. $s_{\alpha}=\operatorname{dim}_{\mathbb{K}} S_{\alpha}$.

Lemma 2.1. For $l \gg 0$ and $D \in \mathrm{Cl}\left(X_{\Sigma}\right)$,

$$
\chi\left(\mathcal{O}_{X}(D)\right)=\sum_{i=0}^{n}(-1)^{i} h^{i}(D)=s_{D}-\sum_{i=0}^{n+1}(-1)^{i} \operatorname{ext}_{S}^{i}\left(S / B(\Sigma)^{[l]}, S(D)\right)_{0} .
$$

Proof. $\operatorname{Ext}_{S}^{0}\left(S / B(\Sigma)^{[l]}, S\right)=\operatorname{Ext}_{S}^{1}\left(S / B(\Sigma)^{[l]}, S\right)=0$, so this follows from [6].

Lemma 2.2. If $F_{\bullet}$ is a free resolution for $S / B(\Sigma)^{[l]}$, then

$$
\begin{aligned}
\sum_{i=0}^{n+1}(-1)^{i} \operatorname{ext}_{S}^{i}\left(S / B(\Sigma)^{[l]}, S(D)\right)_{0} & =\sum_{i=0}^{d}(-1)^{i} \operatorname{dim}_{\mathbb{K}} F_{i}^{\vee}(D)_{0} \\
& =\sum_{i=0}^{d}(-1)^{i} \operatorname{dim}_{\mathbb{K}}\left(F_{i}\right)_{D}^{\vee} .
\end{aligned}
$$

Proof. Take Euler characteristics.

Lemma 2.3. If $F_{\bullet}$ is a minimal free resolution for $S / B(\Sigma)^{[l]}$, then

$$
\operatorname{dim}_{\mathbb{K}}\left(F_{i}\right)_{D}^{\vee}=\sum_{D^{\prime} \in \operatorname{Cl}\left(X_{\Sigma}\right)} \operatorname{tor}_{i}^{S}\left(S / B(\Sigma)^{[l]}, \mathbb{K}\right)_{D^{\prime}} \cdot s_{D^{\prime}+D} .
$$

Proof. Let $F_{\bullet}$ be a minimal free resolution for $S / B(\Sigma)^{[l]}$, and

$$
r_{i}\left(D^{\prime}\right)=\operatorname{tor}_{i}^{S}\left(S / B(\Sigma)^{[l]}, \mathbb{K}\right)_{D^{\prime}} .
$$

Then

$$
F_{i}=\bigoplus_{D^{\prime} \in \operatorname{Cl}\left(X_{\Sigma}\right)} S\left(-D^{\prime}\right)^{r_{i}\left(D^{\prime}\right)} .
$$

Now dualize and take the shift by $D$ into account. 


\section{Combinatorial commutative algebra}

Taylor resolution. We now observe that the multigraded betti numbers $r_{i}\left(D^{\prime}\right)$ of $S / B(\Sigma)^{[l]}$ can be replaced with related numbers which arise from a Taylor resolution for $S / B(\Sigma)$. The Taylor resolution 23 of a monomial ideal is a variant of the Koszul complex, which takes into account the LCM's of the monomials involved.

Let $I=\left\langle m_{1}, \ldots, m_{k}\right\rangle$ be a monomial ideal, and consider a complete simplex with vertices labelled by the $m_{i}$, and each $n$-face $F$ labelled with the LCM of the $n+1$ monomials corresponding to vertices of $F$. Define a chain complex where the differential on an $n$-face $F=\left[v_{i_{0}}, \ldots, v_{i_{n}}\right]$ is

$$
d(F)=\sum_{j=0}^{n}(-1)^{j} \frac{m_{F}}{m_{F \backslash v_{i_{j}}}} F \backslash v_{i_{j}},
$$

with $m_{F}$ denoting the monomial labelling face $F$. As shown by Taylor, this complex is actually a resolution (though often nonminimal) of $I$. When the $m_{i}$ are squarefree, the LCM of a subset of $l^{t h}$ powers is the $l^{\text {th }}$ power of the LCM of the original monomials, hence the Taylor resolution for $I^{[l]}$ is given by the $l^{\text {th }}$ power of the Taylor resolution for $I$, in the sense that a summand $S(-\alpha)$ in the free resolution for $I$ is replaced with $S(-l \cdot \alpha)$ in the resolution for $I^{[l]}$.

Thus, the Taylor resolution of $S / B(\Sigma)$ determines the Taylor resolution of $S / B(\Sigma)^{[l]}$. The formula in Lemma 2.3 requires a minimal free resolution, which the Taylor resolution is generally not. However, this is no obstacle:

Lemma 3.1. If $F_{\bullet}$ is a free resolution for $S / B(\Sigma)$, then

$\sum_{i=0}^{n+1}(-1)^{i} \operatorname{ext}_{S}^{i}\left(S / B(\Sigma)^{[l]}, S(D)\right)_{0}=\sum_{i=0}^{d}(-1)^{i} \sum_{D^{\prime} \in \mathrm{Cl}\left(X_{\Sigma}\right)} \operatorname{tor}_{i}^{S}(S / B(\Sigma), \mathbb{K})_{D^{\prime}} \cdot s_{l \cdot D^{\prime}+D}$

Proof. If $F_{\bullet}$ is a minimal resolution of $S / B(\Sigma)^{[l]}$, then Lemmas 2.2 and 2.3 yield

$$
\sum_{i=0}^{n+1}(-1)^{i} \operatorname{ext}_{S}^{i}\left(S / B(\Sigma)^{[l]}, S(D)\right)_{0}=\sum_{i=0}^{d}(-1)^{i} \sum_{D^{\prime} \in \mathrm{Cl}\left(X_{\Sigma}\right)} \operatorname{tor}_{i}^{S}\left(S / B(\Sigma)^{[l]}, \mathbb{K}\right)_{D^{\prime}} \cdot s_{D^{\prime}+D}
$$

Lemma 2.2 shows that the $l^{\text {th }}$ power of a Taylor resolution for $S / B(\Sigma)$ can be used to compute the left-hand side. Furthermore, when $F_{\bullet}$ is non-minimal, in the expression

$$
\sum_{i=0}^{d}(-1)^{i} \operatorname{dim}_{\mathbb{K}}\left(F_{i}\right)_{D}^{\vee}
$$

the nonminimal summands cancel out, hence we may pass back to the description in terms of Tor, yielding the result.

Alexander duality and monomial ideals. Let $\Delta$ be a simplicial complex on vertex set $\{1, \ldots, d\}$. Let $S=\mathbb{Z}\left[x_{1}, \ldots, x_{d}\right]$ be a polynomial ring, with variables corresponding to the vertices of $\Delta$.

Definition 3.2. The Stanley-Reisner ideal $I_{\Delta} \subseteq S$ is the ideal generated by all monomials corresponding to nonfaces of $\Delta$ :

$$
\left.I_{\Delta}=\left\langle x_{i_{1}} \cdots x_{i_{k}}\right|\left[i_{1}, \ldots, i_{k}\right] \text { is not a face of } \Delta\right\rangle .
$$




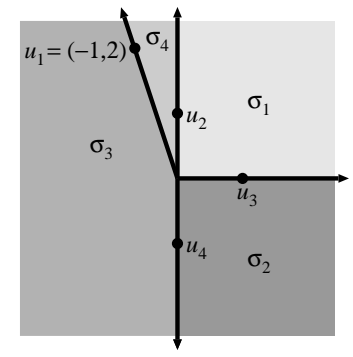

Figure 1. The fan for $\mathcal{H}_{2}$

The Stanley-Reisner ring is $S / I_{\Delta}$. The intersection of a complete simplicial fan $\Sigma \subseteq \mathbb{R}^{n}$ with the unit sphere $S^{n-1}$ gives a simplicial complex we denote by $P_{\Sigma}$; define $I_{\Sigma}$ as the Stanley-Reisner ideal of $P_{\Sigma}$.

Definition 3.3. If $\Delta$ is a simplicial complex on $[d]=\{1, \ldots d\}$, then the Alexander dual $\Delta^{\vee}$ is a simplicial complex consisting of the complements of the nonfaces of $\Delta$ :

$$
\Delta^{\vee}=\{[d] \backslash \sigma \mid \sigma \notin \Delta\} .
$$

Example 3.4. The Hirzebruch surface $\mathcal{H}_{2}$ corresponds to the fan in Figure 1. Since $\left[u_{2}, u_{4}\right]$ and $\left[u_{1}, u_{3}\right]$ are nonfaces of $\Sigma$, and every other nonface such as $\left[u_{1}, u_{2}, u_{4}\right]$ contains them, the Stanley-Reisner ideal is

$$
I_{\Sigma}=\left\langle x_{1} x_{3}, x_{2} x_{4}\right\rangle .
$$

The Alexander dual $\Sigma^{\vee}$ contains all $\rho \in \Sigma(1)$. Since $\widehat{u_{1} u_{3}}=\left[u_{2}, u_{4}\right]$ and $\widehat{u_{2} u_{4}}=$ $\left[u_{1}, u_{3}\right], \Sigma^{\vee}(2)=\left\{\left[u_{2}, u_{4}\right],\left[u_{1}, u_{3}\right]\right\}$. So

$$
I_{\Sigma \vee}=\left\langle x_{1} x_{2}, x_{1} x_{4}, x_{2} x_{3}, x_{3} x_{4}\right\rangle .
$$

Lemma 3.5. The toric irrelevant ideal $B(\Sigma)$ is Alexander dual to the StanleyReisner ideal $I_{\Sigma}$.

Proof. The Alexander dual $I_{\Sigma \vee}$ to $I_{\Sigma}$ is obtained by monomializing ([15], Proposition 1.35) a primary decomposition for $I_{\Sigma}$. If $M C(\Sigma)$ denotes the set of minimal cofaces of $\Sigma$, then the primary decomposition of $I_{\Sigma}$ is

$$
I_{\Sigma}=\bigcap_{\left[i_{1}, \ldots, i_{k}\right] \in M C(\Sigma)}\left\langle x_{i_{1}}, \ldots, x_{i_{k}}\right\rangle .
$$

The ideal $I_{\Sigma^{\vee}}$ is generated by monomials corresponding to minimal cofaces, which are complements to maximal faces, hence $I_{\Sigma^{\vee}}=B(\Sigma)$.

Theorem 3.6 (Danilov [5], Jurkiewicz [10]). For a complete simplicial fan $\Sigma$, let $J=\left\langle\operatorname{div}\left(\chi^{\mathbf{m}}\right) \mid \mathbf{m} \in M\right\rangle$. The rational Chow ring $C h\left(X_{\Sigma}\right)$ is the rational StanleyReisner ring of $\Sigma$, modulo $J$.

The ideal $J$ is minimally generated by a regular sequence; it is these linear forms which encode the geometry of $\Sigma$. To interpret the Euler characteristic of $\mathcal{O}_{X}(D)$ in terms of intersection theory, we must change computations involving the toric irrelevant ideal into computations involving the Stanley-Reisner ideal. For a polynomial ring $R=\mathbb{K}\left[x_{1}, \ldots, x_{d}\right]$ endowed with the fine (also called $\mathbb{Z}^{d}$ ) grading 
$\operatorname{deg}\left(x_{i}\right)=\mathbf{e}_{i} \in \mathbb{Z}^{d}$ and squarefree monomial ideal $M$, the following result of Mustaţă [[16], Corollary 3.1] provides the bridge:

$$
\operatorname{Tor}_{i}^{R}\left(M^{\vee}, \mathbb{K}\right)_{\mathbf{m}} \simeq \operatorname{Ext}_{R}^{|\mathbf{m}|-i}(R / M, R)_{-\mathbf{m}} \text { if } \mathbf{m} \in\{0,1\}^{d}, \text { else } 0 .
$$

Letting $Z=\{0,1\}^{d}$, applying Mustaţă's result yields:

$$
\begin{aligned}
\operatorname{tor}_{i}^{S}(S / B(\Sigma), \mathbb{K})_{D^{\prime}} & =\sum_{\substack{\mathbf{m} \in Z, \phi(\mathbf{m})=D^{\prime}}} \operatorname{tor}_{i}^{S}(S / B(\Sigma), \mathbb{K})_{\mathbf{m}} \\
& =\sum_{\substack{\mathbf{m} \in Z, \phi(\mathbf{m})=D^{\prime}}} \operatorname{ext}_{S}^{|\mathbf{m}|-i+1}\left(S / I_{\Sigma}, S\right)_{-\mathbf{m}}
\end{aligned}
$$

Lemma 3.7. For a complete fan $\Sigma \subseteq N \otimes_{\mathbb{Z}} \mathbb{R} \simeq \mathbb{R}^{n}$ with $|\Sigma(1)|=d$,

(1) $\operatorname{Ext}_{S}^{j}\left(S / I_{\Sigma}, S\right)=0$ for all $j \neq d-n$.

(2) In the $\mathbb{Z}^{d}$ grading, $\operatorname{Ext}_{S}^{d-n}\left(S / I_{\Sigma}, S\right) \simeq S / I_{\Sigma}(\mathbf{1})$.

Proof. From Definition 3.2 $I_{\Sigma}$ is the Stanley-Reisner ideal of the simplicial sphere $P_{\Sigma}$, which is Gorenstein by Corollary II.5.2 of [22]. Since $\operatorname{dim} P_{\Sigma}=n-1$,

$$
\operatorname{codim}\left(I_{\Sigma}\right)=(d-1)-(n-1)=d-n .
$$

Everything follows from this, save that $S / I_{\Sigma}$ is shifted by $\mathbf{1}$. The Gorenstein property means the minimal free resolution of $S / I_{\Sigma}$ is of the form

$$
0 \longrightarrow S(-\alpha) \stackrel{\partial_{d-n}}{\longrightarrow} \bigoplus_{j=1}^{k} S\left(-\beta_{j}\right) \stackrel{\partial_{d-n}-1}{\longrightarrow} \cdots \longrightarrow \bigoplus_{j=1}^{k} S\left(-\gamma_{j}\right) \stackrel{\left[I_{\Sigma}\right]}{\longrightarrow} S \longrightarrow S / I_{\Sigma} \longrightarrow 0
$$

where $\partial_{d-n}$ is (up to signs) the transpose of the matrix of minimal generators $\left[I_{\Sigma}\right]$. To show that the shift in $E x t^{d-n}$ is 1, we use a result of Hochster. For a complex $\Delta$ and weight $\alpha$, let $\left.\Delta\right|_{\alpha}=\{\sigma \in \Delta \mid \sigma \subseteq \alpha\}$. Equating the multidegree 1 with the full simplex on all vertices of $\Delta$, Hochster's formula (5.12 of [15]) yields

$$
\operatorname{Tor}_{d-n}^{S}\left(S / I_{\Sigma}, \mathbb{K}\right)_{\mathbf{1}}=\widetilde{H}^{n-1}\left(\left.\Sigma\right|_{\mathbf{1}}, \mathbb{K}\right)
$$

Since $\left.\Sigma\right|_{1} \simeq P_{\Sigma} \simeq S^{n-1}$, the result follows.

Example 3.8. The Stanley-Reisner ring for the fan $\Sigma$ of Example 3.4 has a $\mathbb{Z}^{4}$ graded minimal free resolution

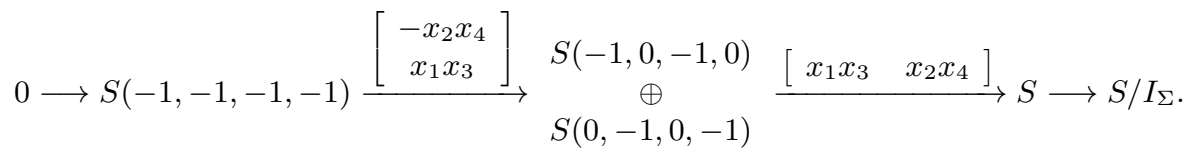

Thus, $\operatorname{Ext}^{2}\left(S / I_{\Sigma}, S\right) \simeq S(1,1,1,1) / I_{\Sigma}$. The simplicial complex $P_{\Sigma}$ consists of vertices [1], [2], [3], [4] and edges [12], [23], [34], [41] and is homotopic to $S^{1}$. Since the multidegrees are all smaller than 1 in the pointwise order, $\left.\Sigma\right|_{1}=P_{\Sigma}$, so

$$
\mathbb{K}=\widetilde{H}^{1}\left(S^{1}, \mathbb{K}\right)=\widetilde{H}^{1}\left(\left.\Sigma\right|_{\mathbf{1}}, \mathbb{K}\right)=\operatorname{Tor}_{2}^{S}\left(S / I_{\Sigma}, \mathbb{K}\right)_{\mathbf{1}},
$$

showing the shift $\alpha$ in the last step of the free resolution of $S / I_{\Sigma}$ is $S(-\mathbf{1})$. 


\section{Proof of Equation 1}

We now prove Equation 1, By Equation 5 ,

$$
\chi\left(\mathcal{O}_{X}(D)\right)=s_{D}-\sum_{i=0}^{n+1}(-1)^{i} \operatorname{ext}_{S}^{i}\left(S / B(\Sigma)^{[l]}, S(D)\right)_{0} .
$$

Let $\gamma(\mathbf{m})=s_{l \cdot \phi(\mathbf{m})+D}$ and $E=\sum_{i=0}^{n+1}(-1)^{i} e x t_{S}^{i}\left(S / B(\Sigma)^{[l]}, S(D)\right)_{0}$. It suffices to show

$$
E=s_{D}+\sum_{\mathbf{m} \in Z \backslash \mathbf{0}}(-1)^{|\mathbf{m}|-d+n+1} \operatorname{dim}_{\mathbb{K}}\left(S / I_{\Sigma}\right)_{\mathbf{1}-\mathbf{m}} \cdot \gamma(\mathbf{m}) .
$$

First, observe that

$$
\begin{aligned}
E & =\sum_{i=0}^{d}(-1)^{i} \sum_{D^{\prime} \in \operatorname{Cl}\left(X_{\Sigma}\right)}\left(\sum_{\substack{\mathbf{m} \in Z, \phi(\mathbf{m})=D^{\prime}}} \operatorname{tor}_{i}^{S}(S / B(\Sigma), \mathbb{K})_{\mathbf{m}}\right) \cdot \gamma(\mathbf{m}) . \\
& =\sum_{i=0}^{d}(-1)^{i} \sum_{\mathbf{m} \in Z} \operatorname{tor}_{i}^{S}(S / B(\Sigma), \mathbb{K})_{\mathbf{m}} \cdot \gamma(\mathbf{m}) . \\
& =s_{D}+\sum_{i=1}^{d}(-1)^{i} \sum_{\mathbf{m} \in Z \backslash \mathbf{0}} \operatorname{tor}_{i}^{S}(S / B(\Sigma), \mathbb{K})_{\mathbf{m}} \cdot \gamma(\mathbf{m}) .
\end{aligned}
$$

The first line follows from Lemma 3.1, the second line is simply a rearrangement, and the third line follows from the observation that

$$
s_{D}=\operatorname{tor}_{0}^{S}(S / B(\Sigma), \mathbb{K})_{\mathbf{0}} \cdot \gamma(\mathbf{0}) .
$$

For $i \geq 0$,

$$
\operatorname{Tor}_{i}^{S}(B(\Sigma), \mathbb{K}) \simeq \operatorname{Tor}_{i+1}^{S}(S / B(\Sigma), \mathbb{K}),
$$

so using Equation 7 we may rewrite the last line of Equation 9 as

$$
s_{D}+\sum_{i=0}^{d-1}(-1)^{i+1} \sum_{\mathbf{m} \in Z \backslash \mathbf{0}} \operatorname{ext}_{S}^{|\mathbf{m}|-i}\left(S / I_{\Sigma}, S\right)_{-\mathbf{m}} \cdot \gamma(\mathbf{m}) .
$$

By Lemma 3.7 $\operatorname{Ext}_{S}^{|\mathbf{m}|-i}\left(S / I_{\Sigma}, S\right)$ is nonzero iff $|\mathbf{m}|-i=d-n$, and

$$
\operatorname{Ext}_{S}^{d-n}\left(S / I_{\Sigma}, S\right) \simeq S / I_{\Sigma}(\mathbf{1}) \text {. }
$$

Since the only nonzero terms in Equation 10 occur for $i=|\mathbf{m}|-d+n$ we rewrite Equation 10 as

$$
\begin{aligned}
& =s_{D}+\sum_{\mathbf{m} \in Z \backslash \mathbf{0}}(-1)^{|\mathbf{m}|-d+n+1} \operatorname{ext}_{S}^{d-n}\left(S / I_{\Sigma}, S\right)_{-\mathbf{m}} \cdot \gamma(\mathbf{m}) \\
& =s_{D}+\sum_{\mathbf{m} \in Z \backslash \mathbf{0}}(-1)^{|\mathbf{m}|-d+n+1} \operatorname{dim}_{\mathbb{K}}\left(S / I_{\Sigma}\right)_{\mathbf{1}-\mathbf{m}} \cdot \gamma(\mathbf{m})
\end{aligned}
$$

This shows that

$$
E=s_{D}+\sum_{\mathbf{m} \in Z \backslash \mathbf{0}}(-1)^{|\mathbf{m}|-d+n+1} \operatorname{dim}_{\mathbb{K}}\left(S / I_{\Sigma}\right)_{\mathbf{1}-\mathbf{m}} \cdot \gamma(\mathbf{m}),
$$

and Equation 1 follows. 
Example 4.1. Consider the divisor $D=3 D_{3}-5 D_{4}$ on the Hirzebruch surface $\mathcal{H}_{2}$ from Figure 1. Since the support function for $D$ is not convex, $D$ is not nef. Thus, computing $\chi\left(\mathcal{O}_{\mathcal{H}_{2}}(D)\right)$ involves more than a simple global section computation. A direct calculation with Riemann-Roch for surfaces shows that

$$
\chi\left(\mathcal{O}_{\mathcal{H}_{2}}(D)\right)=4 .
$$

Using the methods of $\S 9.4$ of [4, it can be shown that $h^{0}(D)=0, h^{1}(D)=2$, and $h^{2}(D)=6$. Now we illustrate how to apply Equation 1 , Let

$$
\phi=\left(\begin{array}{cccc}
1 & -2 & 1 & 0 \\
0 & 1 & 0 & 1
\end{array}\right)
$$

so that the Class group is given by

$$
\mathbb{Z}^{4} \stackrel{\phi}{\longrightarrow} \mathbb{Z}^{2} \simeq C l\left(\mathcal{H}_{2}\right) \longrightarrow 0
$$

The Eisenbud-Mustaţă-Stillman bound of Equation 4 is $l=80$, but a careful analysis (see Example 3.6 of [6]) shows that in this case taking $l=4$ is sufficient. Then for example with $\mathbf{m}=(0,1,0,1)$ we have $\phi(\mathbf{m})=(-2,2)$ so since $D=(3,-5)$,

$$
S_{4 \cdot \phi(\mathbf{m})+D}=S_{(-5,3)}=H^{0}\left(\mathcal{O}_{\mathcal{H}_{2}}(-5,3)\right),
$$

and the dimension of this space is two. However,

$$
\left(S / I_{\Sigma}\right)_{1-(0,1,0,1)}=\left(S / I_{\Sigma}\right)_{(1,0,1,0)}=0,
$$

since $x_{1} x_{3} \in I_{\Sigma}$. A check shows that all terms in the summation vanish, save when

$$
\mathbf{m} \in\{(1,1,0,1),(0,1,1,1),(1,1,1,1)\}
$$

For the first two values, $\phi(\mathbf{m})=(-1,2)$, and we compute

$$
S_{4 \cdot \phi(\mathbf{m})+D}=S_{(-1,3)}=H^{0}\left(\mathcal{O}_{\mathcal{H}_{2}}(-1,3)\right),
$$

which has dimension twelve. Since $\mathbf{1}-\mathbf{m}$ is either $(0,0,1,0)$ or $(1,0,0,0)$, for these two values of $\mathbf{m}$,

$$
\operatorname{dim}_{\mathbb{K}}\left(S / I_{\Sigma}\right)_{\mathbf{1}-\mathbf{m}}=1
$$

Since $|\mathbf{m}|-d+n=1$, these two weights contribute $(-1) \cdot 2 \cdot 12=-24$ to the Euler characteristic. For the remaining weight $\mathbf{m}=(1,1,1,1)$, the Stanley-Reisner ring is one dimensional in degree $\mathbf{1}-\mathbf{m}=(0,0,0,0)$, and $\phi(1,1,1,1)=(0,2)$ and

$$
S_{4 \cdot \phi(\mathbf{m})+D}=S_{(3,3)}=H^{0}\left(\mathcal{O}_{\mathcal{H}_{2}}(3,3)\right),
$$

which has dimension 28. Since $|\mathbf{m}|-d+n=2$ the contribution is positive, thus

$$
\chi\left(\mathcal{O}_{\mathcal{H}_{2}}\left(3 D_{3}-5 D_{4}\right)\right)=-24+28=4 .
$$

Problem As noted in the introduction, this work began as an attempt to find a toric proof of Hirzebruch-Riemann-Roch using Equation 1 it would be interesting to find such a proof. A proof of Equation 1 also follows from results of MaclaganSmith [14, I thank Greg Smith for noting this.

Acknowledgements Computations were performed using Macaulay2 8 by Grayson and Stillman, and NormalToricVarieties 21] by Greg Smith. 


\section{REFERENCES}

[1] R. Blumenhagen, B. Jurke, T. Rahn, H. Roschy, Cohomology of line bundles: A computational algorithm, J. Math. Phys. 51, 103525 (2010)

[2] M. Brion, M. Vergne, An equivariant Riemann-Roch theorem for complete, simplicial toric varieties, J. Reine Angew. Math. 482 (1997), 67-92.

[3] D. Cox, The homogeneous coordinate ring of a toric variety, J. Algebraic Geom. 4 (1995), $15-50$.

[4] D. Cox, J. Little and H. Schenck, Toric Varieties, AMS Graduate Studies in Mathematics, to appear, available at http://www.cs.amherst.edu/ dac/toric.html

[5] V. Danilov, The geometry of toric varieties, Russian Math. Surveys 33 (1978), 97-154; Uspekhi Mat. Nauk. 33 (1978), 85-134.

[6] D. Eisenbud, M. Mustaţă, and M. Stillman, Cohomology on toric varieties and local cohomology with monomial supports, J. Symbolic Comput. 29 (2000), 583-600.

[7] W. Fulton, Introduction to Toric Varieties, Princeton Univ. Press, Princeton, NJ, 1993.

[8] D. Grayson and M. Stillman, Macaulay2, available at http://www.math.uiuc.edu/Macaulay2.

[9] S. Jow, Cohomology of toric line bundles via simplicial Alexander duality, arXiv:1006.0780.

[10] J. Jurkiewicz, Chow ring of projective nonsingular torus embedding, Colloq. Math. 43 (1980), $261-270$

[11] A.G. Khovanskii, Newton polyhedra, and toroidal varieties, Funkcional. Anal. i Prilozen. 11 (1977), 56-64.

[12] A.G. Khovanskii, A.V. Pukhlikov, Finitely additive measures of virtual polyhedra, Algebra i Analiz 4 (1992), 161-185.

[13] A.G. Khovanskii, A.V. Pukhlikov, The Riemann-Roch theorem for integrals and sums of quasipolynomials on virtual polytopes, Algebra i Analiz 4 (1992), 188-216.

[14] D. Maclagan, G.G. Smith, Multigraded Castelnuovo-Mumford Regularity, J. Reine Angew. Math. 571 (2004), 179-212.

[15] E. Miller, B. Sturmfels, Combinatorial commutative algebra, Graduate Texts in Mathematics, vol. 227, Springer-Verlag, Berlin-Heidelberg-New York, 2005.

[16] M. Mustață, Local cohomology at monomial ideals, J. Symbolic Comput. 29 (2000), 709-720.

[17] M. Mustaţă, Vanishing theorems on toric varieties, Tohôku Math. J. 54 (2002), 451-470.

[18] J. Pommersheim, Products of cycles and the Todd class of a toric variety, J. Amer. Math. Soc. 9 (1996), 813-826.

[19] J. Pommersheim, H. Thomas, Cycles representing the Todd class of a toric variety, J. Amer. Math. Soc. 17 (2004), 983-994.

[20] T. Rahn, H. Roschy, Cohomology of line bundles: Proof of the Algorithm, J. Math. Phys. 51, $103520(2010)$

[21] G. G. Smith, NormalToricVarieties, available at http://www.math.uiuc.edu/Macaulay2.

[22] R. Stanley, Combinatorics and commutative algebra, Birkhäuser, Boston, MA, 1996.

[23] D. Taylor, Ideals generated by monomials in an R-sequence, Thesis, Univ. Chicago (1966).

Schenck: Mathematics Department, University of Illinois, Urbana, IL 61801, USA

E-mail address: schenck@math.uiuc.edu 